\title{
Evacuación del meconio intestinal para mejorar tolerancia alimentaria en prematuro de muy bajo peso (protocolo Emita)
}

\author{
PATRICIA MENA N. ${ }^{1}$, JORGE LEÓN DEL P. ${ }^{2}$, DANIELA SANDINO P. ${ }^{3}$, \\ PAMELA RALMOLFO B. ${ }^{3}$, DEBORA SABATELLI ${ }^{4}$, ADOLFO LLANOS M. ${ }^{5}$, \\ BEATRIZ MILET L. ${ }^{1}$, RED NEONATAL NEOCOSUR \\ 1. Hospital Dr. Sótero del Río, Puente Alto. \\ 2. Hospital Guillermo Grant Benavente, Concepción. \\ 3. Hospital Gustavo Fricke, Viña del Mar, Chile. \\ 4. Hospital Juan A. Fernández, Buenos Aires. \\ 5. Miami Children's Hospital, Miami, EEUU.
}

\begin{abstract}
Meconium evacuation to improve feeding tolerance in very low birth weight preterm infants (Emita Protocol)
\end{abstract}

Introduction: It has been reported that feeding tolerance in preterm infants is associated with an early passage of meconium. Prospective, randomized or historical control studies that stimulate meconium evacuation have reported varied results. This study was intented to evaluate the use of enemas to speed up meconium evacuation, facilitating feeding tolerance. Patients and Method: A controlled multicenter randomized trial that evaluated the use of physiological saline enemas with glycerol $(0.8 \mathrm{ml}$ glycerol $+3 \mathrm{ml}$ saline or $1 \mathrm{ml}$ glycerol $+5 \mathrm{ml}$ saline depending on babies weighing less or more than $800 \mathrm{~g}$ at birth, respectively) versus simulation. This procedure was performed in the first 96 hours of life in infants with birth weight between 500 and 1,250 g. Maternal (preterm delivery, clinical chorioamnionitis, gestational hypertension, administration of magnesium sulfate and prenatal corticosteroids, fetal Doppler altered, type of delivery, gender, weight and gestational age, assessment of Apgar and need for assisted ventilation and oxygenotherapy) and nutritional history (age when feeding volumes of $100 \mathrm{ml} / \mathrm{kg}$ /day and full enteral feeding were reached, age to remove meconium, number of days on parenteral nutrition, weight at 28 days, weekly volumes of breast milk and preterm formula) were described. Results: No significant differences were obtained regarding the age to reach full enteral intake or 100 $\mathrm{ml} / \mathrm{kg} /$ day were found among the 101 patients in the study. Also, no differences in the following secondary variables are observed: number of episodes of late sepsis with or without positive blood culture, hyperbilirubine-

Recibido el 29 de julio de 2013, devuelto para corregir el 11 de octubre de 2013, segunda versión 04 de noviembre de 2013 , aceptado para publicación el 18 de marzo de 2014.

Este estudio fue parcialmente financiado por el Concurso de Proyectos de Investigación de la Rama de Neonatología de la Sociedad Chilena de Pediatría 2010.

Este trabajo cumple con los requisitos sobre consentimiento /asentimiento informado, comité de ética, financiamiento, estudios animales y sobre la ausencia de conflictos de intereses según corresponda.

Correspondencia a:

Patricia Mena N.

E-mail: pmenanani@gmail.com 
mia, necrotizing enterocolitis and intraventricular hemorrhage. Conclusions: The routine use of saline enemas and glycerin in this study does not alter the enteral feeding tolerance in very low birth weight preterm infants. (Key words: Enema, tolerance, enteral feeding, meconium, very low birth weight).

Rev Chil Pediatr 2014; 85 (3): 304-311

\section{RESUMEN}

Introducción: Se ha descrito que la tolerancia alimentaria en el extremo prematuro se asocia a una precoz eliminación de meconio. Estudios prospectivos, randomizados o con controles históricos de estimulación de evacuación de meconio reportan diferentes resultados. El objetivo de este estudio fue evaluar el uso sistemático de enemas que aceleren la evacuación de meconio, y por ende la tolerancia alimentaria. Pacientes y Método: Estudio controlado, aleatorizado y multicéntrico que evaluó el uso de enemas de solución fisiológica con glicerina $(0,8 \mathrm{ml}$ de glicerina $+3 \mathrm{ml}$ de solución fisiológica o $1 \mathrm{ml}$ de glicerina $+5 \mathrm{ml}$ de solución fisiológica según peso al nacer menor o mayor de $800 \mathrm{~g}$, respectivamente) versus simulación, iniciado en los primeras $96 \mathrm{~h}$ de vida, en prematuros con peso al nacimiento entre 500 y $1.250 \mathrm{~g}$. Se registraron antecedentes maternos (parto prematuro, infección ovular, síndrome hipertensivo del embarazo, administración de sulfato de magnesio, y corticoides prenatales, doppler fetal alterado, tipo de parto, sexo, peso y edad gestacional, evaluación de apgar y necesidad de ventilación asistida y oxígenoterapia) y nutricionales (edad al alcanzar volúmenes de alimentación de $100 \mathrm{ml} / \mathrm{kg} /$ día y alimentación enteral completa, edad para eliminar meconio, número de días en nutrición parenteral, peso a los 28 días, volúmenes semanales de leche materna y fórmula de prematuros). Resultados: En 101 sujetos incluidos en el estudio, no se obtienen diferencias significativas en la variable principal de edad para alcanzar el aporte enteral total o los $100 \mathrm{ml}$ por kg por día. Tampoco se observan diferencias en las variables secundarias: número de episodios de sepsis tardías con o sin hemocultivo positivo, hiperbilirrubinemia, enterocolitis necrotizante o hemorragia intracraneana. Conclusiones: El uso sistemático de enemas de solución fisiológica y glicerina, en la forma utilizada en este estudio, no modifica la tolerancia alimentaria enteral de prematuros de muy bajo peso al nacer.

(Palabras clave: Enema, tolerancia, aporte enteral, meconio, muy bajo peso de nacimiento).

Rev Chil Pediatr 2014; 85 (3): 304-311

\section{Introducción}

El recién nacido de muy bajo peso de nacimiento (peso al nacer $<1.500 \mathrm{~g}, \mathrm{RNMBPN}$ ) presenta con gran frecuencia problemas de tolerancia alimentaria, caracterizados por incremento en los residuos alimentarios, distensión abdominal asociada a demora en la eliminación de meconio, y en algunos casos a impacto de meconio a nivel intestinal. Estas dificultades determinan que la alimentación por vía enteral se inicia tardíamente o se suspende con frecuencia, no logrando aportes significativos por vía enteral en las primeras semanas de vida. Esta situación es más prevalente en los niños de extremo bajo peso (peso al nacer $<$ $1.000 \mathrm{~g}$, RNEBPN), en los pequeños para edad gestacional (PEG) y en los hijos de madre hipertensa, especialmente si ha sido tratada con sulfato de magnesio ${ }^{1-4}$. Como consecuencia de la demora en establecer la alimentación enteral, los prematuros requieren más tiempo de nutrición parenteral incrementando el riesgo de infecciones ${ }^{5,6}$, enterocolitis necrotizante $\left(\mathrm{ECN}^{7,8}\right)$, perforación intestinal ${ }^{2}$, y colestasia, comprometiendo además el aporte de nutrientes con consecuencias desfavorables en el crecimiento y neurodesarrollo de esta población ${ }^{9}$.

Ha sido postulado que la falta de eliminación y movilización del meconio en estos recién nacidos puede conducir a sobredistensión de asas intestinales como consecuencia de una obstrucción meconial no relacionada a fibrosis quística ${ }^{2}$. El análisis anatomopatológico de perforaciónes intestinales aisladas en prematuros extremos sugiere que la sobredistensión producida por un tapón meconial produce un adelgazamiento de la muscular de la mucosa con perforación única precoz o favorece la presentación tardía de una enterocolitis al de- 
jar un segmento con menor motilidad y mayor riesgo de sobreproliferación bacteriana ${ }^{7,8}$.

Las conductas clínicas frente a esta situación son muy variables e incluyen desde una conducta expectante, hasta la necesidad de movilizar el meconio en situaciones de distensión marcada, ya sea mediante el uso de enema con gastrograffin o laparotomía exploratoria en los casos más severos ${ }^{1,2,10}$. Otra aproximación al problema ha sido el uso temprano de enemas con glicerina. Se han publicado dos trabajos, uno controlado randomizado ${ }^{11}$ y otro con control histórico ${ }^{12}$ mostrando diferentes resultados. El metaanálisis del efecto de usar glicerina sobre la tolerancia alimentaria concluye que la evidencia no es suficiente y que es necesario realizar más estudios ${ }^{13}$.

Se planifica un estudio para evaluar la efectividad del uso de enemas evacuantes para mejorar la tolerancia alimentaria de recién nacidos prematuros. El objetivo principal fue evaluar el efecto del uso sistemático de enemas con glicerina y solución fisiológica en la tolerancia alimentaria definida por el número de días para alcanzar aporte enteral de $100 \mathrm{ml}$ por $\mathrm{Kg}$ día y aporte enteral total, que corresponde a la suspensión del aporte de líquidos por vía endovenosa. Los objetivos secundarios fueron evaluar el efecto de los enemas con glicerina y solución fisiológica en la incidencia de sepsis con y sin hemocultivo positivo en los primeros 28 días de vida, en hiperbilirrubinemia, sospecha de enterocolitis necrotizante, exploración quirúrgica abdominal y hemorragia intracraneana, este último como potencial evento adverso asociado al procedimiento.

\section{Pacientes y Método}

\section{Diseño}

El estudio fue experimental, multicéntrico, con asignación aleatoria a un grupo de estudio y un grupo control. Se estratificó por peso de nacimiento: menor de 900 y entre 900 y 1.250 g y por centro, con tabla aleatoria y se entregó sobres cerrados y numerados a cada centro con la asignación de cada caso. Con el objetivo de mantener el ciego en el equipo médico tratante, la asignación de la intervención sólo fue cono- cido por el profesional encargado de realizar o simular el tratamiento, quien no participó en la atención directa del paciente durante sus primeras 6 semanas de vida. El estudio fue aprobado por el comité de ética de las instituciones participantes, y se obtuvo consentimiento informado de los padres o cuidador legal antes del inicio del estudio.

\section{Sujetos}

Candidatos al estudio fueron todos los recién nacidos con peso de nacimiento entre 500 g y 1.250 g admitidos a 4 unidades de cuidado intensivo neonatal pertenecientes a la red Neocosur. Pacientes fueron elegibles si en las primeras $96 \mathrm{~h}$ de vida estaban clínicamente estables definido como normotensos, con o sin vasoactivos, normoxemicos y con saturación mantenida sobre $85 \%$. Se excluyeron pacientes con malformaciones congénitas mayores y del tracto gastrointestinal, quienes desarrollaron hemorragia intaracraneana grado III o más antes de ser aleatorizado, requirieron $\mathrm{FiO}_{2}$ mayor o igual a 0,6 después de las $12 \mathrm{~h}$ de vida, presentaron falla multisistémica, o en quienes el consentimiento informado no se obtuvo en primeras $96 \mathrm{~h}$.

\section{Intervención}

El grupo tratado recibió enema evacuante de glicerina con solución fisiológica, y en el grupo control se simuló el procedimiento. El grupo tratado recibió $0,8 \mathrm{ml}$ de glicerina +3 $\mathrm{ml}$ de solución fisiológica o $1 \mathrm{ml}$ de glicerina + $5 \mathrm{ml}$ de solución fisiológica según peso al nacer menor o mayor de $800 \mathrm{~g}$, respectivamente. La dosis es intermedia de los estudios previos. Los enemas se iniciaron entre las 12 y $96 \mathrm{~h}$ de vida; colocándose cada $12 \mathrm{~h}$ si no presentaba deposiciones, o cada $24 \mathrm{~h}$ si presentaba. Los enemas se continuaron hasta que presentó deposiciones de transición y no meconio, lo que se documentó en registro de enfermería. La administración del enema en el grupo tratado se realizó con el niño en decúbito lateral, evitando la flexión marcada de las piernas sobre el abdomen, introduciendo una sonda $1,5 \mathrm{~cm}$ y colocando con suavidad el enema. La simulación se realizó con el uso de una sonda y jeringa, pero no se coloca a nivel rectal. Se registró 
en ambos casos la colocación del enema en la hoja de enfermería.

Con el fin de estandarizar el manejo nutricional, a los centros participantes se les sugirió inicio de nutrición parenteral temprana (primeras $24 \mathrm{~h}$ de vida) y usar un protocolo de alimentación enteral, que se enmarca en las prácticas habituales con aumentos de volumen de $15 \mathrm{ml}$ por $\mathrm{Kg}$ de peso actual. Se suspendió el aporte enteral según criterios locales de tolerancia alimentaria.

\section{Registro}

Se registraron antecedentes maternos de parto prematuro, infección ovular, sindrome hipertensivo del embarazo (SHE), administración de sulfato de magnesio, y corticoides prenatales, doppler fetal alterado, tipo de parto, sexo, peso y edad gestacional (mejor estimado obstétrico) al nacer, evaluación de apgar y necesidad de ventilación asistida y oxigenoterapia. Con respecto a las variables de tolerancia alimenticia y nutricionales, se registró el número de enemas por semana, la edad al alcanzar volúmenes de alimentación de 100 $\mathrm{ml} / / \mathrm{kg} /$ día y alimentación enteral completa, edad para eliminar meconio, el número de días en nutrición parenteral, el peso a los 28 días, los volúmenes semanales de leche materna y fórmula de prematuros. Las variables clínicas evaluadas incluyeron: sepsis tardía (inicio después del tercer día de vida) con y sin hemocultivo positivo, ésta última tratada con más de 5 días de antibióticos; enterocolitis necrotizante definida como sospecha, con distensión o residuos con suspensión de alimentación, más inicio o cambio de antibióticos pero no confirmada posteriormente; y enterocolitis confirmada con grado II o más, con alteración radiológica; cirugía por perforación intestinal o enterocolitis; hemorragia intracraneana, uso de enema con medio de contrate con diagnostico de ileo meconial.

\section{Cálculo de tamaño muestral}

En base a datos previos de la red Neocosur para este grupo de peso, se estimó que con una disminución del porcentaje de niños que no han alcanzado el aporte enteral total, después de 15 días de iniciado, de $25 \%$ a $12 \%$; se requieren 139 niños por grupo con error alfa de 0,05 y beta de $20 \%$. Después de corregir por mortalidad después de las $48 \mathrm{~h}$ de vida, y retiro eventual del estudio, se estimaron 155 sujetos por grupo. Se planteó cierre del estudio a los 2 años de iniciado, independientemente del tamaño alcanzado, considerando cambios en las prácticas clínicas y eventual vigencia de la pregunta formulada.

\section{Eventos adversos}

Se consideraron eventos adversos serios: rectorragia o hemorragia digestiva, complicaciones quirúrgicas digestivas: laparotomía exploradora, perforación única, enterocolitis necrotizante complicada; y hemorragia intracraneana grado III o IV, muerte de cualquier causa. Estos fueron reportados dentro de las $96 \mathrm{~h}$ de ocurridos, a un comité adjunto.

\section{Análisis estadístico}

Se realizó por intención primaria de tratamiento. Como análisis secundario se analizaron las variables primarias y el número de cuadros sépticos (clínicos o confirmados) en relación al número de enemas realizados. Se utilizó el $\chi^{2}$ para variables categóricas, test de student para continuas paramétricas, las variables no paramétricas, determinadas con la prueba Shapiro-Wilks de normalidad, fueron analizadas con Mann-Whitney Wilcoxon. Se consideró significativo un $\mathrm{p} \leq 0,05$. Se realizó análisis de regresión logística para evaluar la asociación de mejor tolerancia alimentaria con: grupo de estudio, peso de nacimiento, reporte de doppler alterado, número de sepsis tardías con hemocultivo positivo, y uso de más de $50 \%$ de volumen enteral como leche materna.

\section{Resultados}

La población estudiada fue de 101 casos, 51 controles y 50 tratados, ingresados entre el 6 de junio de 2010 y el 30 de junio de 2012. En el hospital Guillermo Grant se ingresaron 41 casos, 51 en el hospital Gustavo Fricke y 9 en el Sanatorio Fernández. En el hospital Sótero del Río se ingresaron 3 pacientes, con los que 
se observó indicación médica de enemas, ya que hacía un año y medio se había incorporado esta práctica clínica por lo que se decidió excluir al centro, ya que no se aseguraba adherencia al protocolo.

La tabla 1 muestra que los dos grupos fueron comparables en características maternas y al nacimiento. Se consideró "riesgo prenatal" la presencia de dos o más de los siguientes antecedentes: síndrome hipertensivo materno, ser PEG o tener un doppler fetal alterado. El grupo que recibió enemas presentó un riesgo prenatal de $60 \%$ comparado con $39 \%$ el grupo control, pero la diferencia no fue estadísticamente significativa.

En la tabla 2 se muestran los resultados de las variables primarias y secundarias analizadas. No hubo ninguna diferencia en las variables de tolerancia alimentaria entre ambos grupos, ni en las variables secundarias como sepsis, enterocolitis, cirugía abdominal, bilirrubina máxima observada, días con deposiciones meconiales. Sólo la sospecha de enterocolitis fue más frecuente en el grupo control. El análisis ajustado por riesgo prenatal muestra similares resultados.

El análisis del subgrupo con más riesgo de intolerancia alimentaria: menores de $800 \mathrm{~g}$ y con algún factor asociado: madre hipertensa, doppler alterado, tampoco mostró ninguna diferencia (datos no se muestran).

En la tabla 3 se muestra el análisis de regresión logística de los factores asociados a no lograr alimentación enteral a los 17 días. Se observa una asociación significativa solamente con haber presentado más sepsis tardías con hemocultivo positivo, sin relación con el grupo de estudio, menor peso de nacimiento, evaluación de flujos umbilicales fetales alterados o aporte de leche materna menor de $50 \%$. La evaluación de eventos adversos reportados no muestra relación directa con el procedimiento de enema.

\section{Discusión}

El estudio no demostró un efecto significativo en mejorar la tolerancia alimentaria con el uso de enemas en la población estudiada. El tamaño muestral planteado para detectar una reducción a la mitad del grupo que no alcanza aporte enteral total a los 15 días no fue logrado. Este cálculo consideró los datos previos de la base del Neocosur para los menores de $1.250 \mathrm{~g}$, pero esta cifra fue de $25 \%$, siendo en esta población mucho mayor, de más de $55 \%$. Con los datos observados en el estudio, se re-

Tabla 1. Antecedentes

\begin{tabular}{|c|c|c|c|}
\hline Grupo & Enema & Control & $\mathbf{p}$ \\
\hline $\mathrm{n}$ & 50 & 51 & \\
\hline Infecc ovular \% & 19,1 & 20,8 & ns \\
\hline Doppler alterado \% & 32 & 21 & ns \\
\hline Parto vaginal \% & 18 & 21,6 & ns \\
\hline SHE \% & 28 & 13,5 & 0,07 \\
\hline Sulfato Mg \% & 30 & 19,2 & ns \\
\hline Edad gest sem* & $27,9(2,1)$ & $27,8(2,0)$ & ns \\
\hline Peso nacimiento $\mathrm{g}^{*}$ & 959 (179) & $957(175)$ & ns \\
\hline Edad Inicio enteral días** & $3(2-21)$ & $3(2-13)$ & ns \\
\hline PEG \% & 42 & 36,5 & ns \\
\hline Corticoides prenatales $* * * \%$ & 54,8 & 46,3 & ns \\
\hline Con riesgo \% & 60,7 & 39,3 & ns \\
\hline
\end{tabular}

*Promedio (desviación estándar. **Mediana (25-75). ${ }^{* * *} 2$ dosis de betametasona prenatal. 
Tabla 2. Resultados

\begin{tabular}{|c|c|c|c|c|c|}
\hline \multirow{3}{*}{$\begin{array}{l}\text { Grupo } \\
\text { Fallecen n (\%) }\end{array}$} & \multirow{3}{*}{$\begin{array}{c}\text { Enema } \\
5(10)\end{array}$} & \multirow{2}{*}{\multicolumn{2}{|c|}{ Control }} & \multicolumn{2}{|c|}{ OR or Coef $(95 \% \mathrm{CI})$ Ajustado $(\mathrm{E} / \mathrm{C})$} \\
\hline & & & & $\mathbf{p}$ & Por riesgo prenatal \\
\hline & & $7(14)$ & ns & 1,04 & $(0,3-3,4)$ \\
\hline Edad Inicio enteral días** & $3(2-5)$ & $3(2-5)$ & ns & 0 & $(-1,3-1,33)$ \\
\hline Edad aporte total días* & $19,3(8,2)$ & $20(10,3)$ & ns & -2 & $(-6,5-2,5)$ \\
\hline Edad $100 \mathrm{ml} / \mathrm{k} / \mathrm{d}$ días* & $15,9(7,9)$ & $16,3(9,6)$ & ns & -1 & $(-4,1-2,2)$ \\
\hline Sin aporte total a 17 días \% & 51.1 & 47,6 & ns & & \\
\hline Bilirrubina max mg/dl* & $7,9(2,3)$ & $7,7(2,3)$ & ns & 0,2 & $(-0,7-1,04)$ \\
\hline Edad con heces trans días* & $12,3(6,3)$ & $12,5(5,5)$ & ns & $-0,2$ & $(-2,7-2,2)$ \\
\hline Días nutrición parenteral & $35,5(32)$ & $36,4(26)$ & ns & $-0,9$ & $(-12-11,2)$ \\
\hline Días nutrición parenteral** & $25(13-48)$ & $23(14-58)$ & ns & & \\
\hline Peso 28 días $g^{*}$ & $1.284(284)$ & $1306(293)$ & ns & 6,2 & $(-6,2-75,3)$ \\
\hline $\begin{array}{l}\text { ECN confirmada } \% \\
p=0,13\end{array}$ & 6 & 15,7 & 0,1 & 0,3 & $(0,08-1,3)$ \\
\hline $\begin{array}{l}\text { Sospecha de ECN \% } \\
p=0,04\end{array}$ & 2 & 13,7 & 0,03 & 0,1 & $(0,01-0,86)$ \\
\hline Cirugía ECN o perforación \%4 & 13,7 & ns & & & \\
\hline Egresa fallecido \% & 10,2 & 14,4 & ns & & \\
\hline CPAP días** & $7,5(2,5-18,5)$ & $10(3-29)$ & ns & & \\
\hline Días con oxígeno** & $17(9-57)$ & $19(10-45)$ & ns & & \\
\hline Días ventilación mecánica** & $3,5(1-17)$ & $5(2-14)$ & ns & & \\
\hline Número enemas sem 1 ** & $5(4-7)$ & $7(5-8)^{* * *}$ & 0,0025 & & \\
\hline HIC \% & 16,7 & 30 & ns & & \\
\hline
\end{tabular}

*Promedio (desviación estándar. ${ }^{* *}$ Medina (25-75). ${ }^{* * *}$ Número de enemas simulados. ECN: Enterocolitis necrotizante. CPAP: Ventilación presión positiva. HIC: Hemorragia intracraneana.

Tabla 3. Regresión logística de alcanzar enteral total antes o después de 17 días

\begin{tabular}{|lcccccc|}
\hline Ent tot 17 & Odds Ratio & Std. Err. & $\mathbf{z}$ & P > z & \multicolumn{1}{|c|}{ [95\% Conf. Interval] } \\
Grupo & 2,267155 & 1,144462 & 1,62 & 0,105 & 0,8429344 & 6,097735 \\
n sepsis hemo & 0,1046311 & 0,0871077 & $-2,71$ & 0,007 & 0,0204652 & 0,5349398 \\
Peso nac g & 1,002355 & 0,0015182 & 1,55 & 0,120 & 0,9993843 & 1,005335 \\
Doppler alt & 0,8786184 & 0,4792426 & $-0,24$ & 0,812 & 0,3016562 & 2,559107 \\
Im_50 & 1,007654 & 0,5159371 & 0,01 & 0,988 & 0,3693863 & 2,748793 \\
\hline
\end{tabular}

Ent: enteral. Nac: nacimiento.

queriría un tamaño muestral de 306 por grupo. Aunque el estudio no permite aclarar si es importante estimular la evacuación de meconio para mejorar la tolerancia alimentaria, si puede contribuir a metaanálisis con otros estudios.

Estudios previos tienen algunas diferencias metodológicas. Heiden y col. ${ }^{11}$, evaluaron el efecto de enemas $(10 \mathrm{ml} / \mathrm{kg}$ de solución fisiológica con $0,8 \mathrm{~g}$ de glicerina) en la evacuación de meconio en 81 RNMBPN aleatoriamente asignados a recibir o no la intervención. Los autores no encontraron diferencias significativas en el tiempo requerido para evacuar meconio.

En el estudio de Shim y col., se comparan un grupo intervenido de 39 RNMBPN quienes recibieron enemas de glicerina de $1 \mathrm{ml}$ en 3 de solución fisiológica por Kilo de peso, desde 
el primer día cada 12 a 24 h, hasta el dia de eliminación total de meconio. El grupo control histórico fue de 44 RNMBPN, en los que se usaba estimulo rectal si no presentaban meconio en 2 a 3 días y en quienes la administración de enema se indicaba sólo si la situación se prolongaba basado en el criterio del médico tratante ${ }^{12}$. Los autores reportan un menor tiempo para alcanzar aporte enteral mayor de 100 $\mathrm{ml} / \mathrm{kg} / \mathrm{dia}$ en el grupo intervenido comparado con el control histórico (mediana $=16$ vs 22,9 ; $\mathrm{p}<0,001)$. El efecto del enema fue mayor para los menores de $1.000 \mathrm{~g}$ de peso de nacimiento (mediana $=17,3$ vs 28,1 días). El estudio también demostró una mayor frecuencia diaria de deposiciones en grupo intervenido en los primeros 5 y 10 días de edad. Igualmente, la duración de uso de catéter central, la incidencia de sepsis tardía y el número de cultivos de catéter positivo fue significativamente menor en el grupo intervenido. El estudio es sugerente de beneficio, pero probablemente no fue la única práctica clínica que puede haber cambiado en un período de 2 años en que transcurren sucesivamente ambas experiencias, con el grupo histórico y el intervenido. Nuestra evaluación usó enemas de un volumen intermedio entre ambos estudios, con una osmolaridad también intermedia. Otro estudio evaluando el uso sistemático de supositorio de glicerina, con 54 casos randomizados, no muestra diferencias significativas pero una tendencia de mejor tolerancia en el grupo tratado ${ }^{14}$.

La preocupación por estimular la eliminación de meconio y mejorar el aporte enteral persiste y un reciente estudio probó el aporte enteral de Gastrografin ${ }^{\circledR}$ diluido ${ }^{15}$, que es una medida previamente utilizada para tratar un taponamiento meconial en prematuros extre$\operatorname{mos}^{10}$. Este estudio aportó Gastrografin ${ }^{\circledR}$ oral $9 \mathrm{ml}$ por kilo en dilución 1:3, en las primeras $24 \mathrm{~h}$ de vida a menores de $1.500 \mathrm{~g}$ o 32 semanas. Aunque hubo poca diferencia en el pasaje de meconio, tanto la tolerancia alimentaria como la duración de la hospitalización en cuidado intensivo, mejoraron significativamente en el grupo tratado; pero, aumentó el riesgo de ECN, lo que puede relacionarse con la alta osmolaridad del Gastrografin ${ }^{\circledR}$ diluido $(717 \mathrm{~m}$ $\mathrm{mOsm} / \mathrm{L})^{15}$.
La clara diferencia en sospecha de enterocolitis reportada en nuestro estudio, sugiere que el uso de enemas reduce la distensión abdominal, por lo que puede tener una indicación individualizada para los pacientes con falta de eliminación de meconio y distención abdominal. Igualmente, el hallazgo de un menor requerimiento de enemas durante la primera semana en el grupo intervenido comparado con el número de enemas simulados en el grupo control sugiere mejoría en la motilidad intestinal con la intervención, considerando que los enemas se indicaban si no existía eliminación espontánea de meconio. A pesar de los beneficios observados en disminuir los episodios de distención abdominal y posible mejoría en motilidad intestinal, el estudio no demostró un efecto en mejorar tolerancia alimentaria. Una posible explicación podría ser el poco poder del tamaño muestral, ya que la mínima diferencia observada requeriría un estudio con una población 7 veces mayor, como se mencionó previamente.

Limitaciones de este estudio: dado que sólo 3 de 20 centros participaron, nuestra población de menores de $1.250 \mathrm{~g}$ puede no ser representativa de la población total del Neocosur. Otros centros tenían otros estudios en marcha o no lograron incorporarse. Varios centros no participaron por la falta de financiamiento de un seguro para los eventuales eventos adversos que se observaran y no fueron autorizados por los Comités de Investigación locales. Además hubo cambios en los investigadores locales que determinaron períodos prolongados sin ingreso de pacientes al estudio. Por otra parte, tampoco se obtuvo que la eliminación de meconio fuera diferente en ambos grupos lo que podría deberse a una estimulación insuficiente, pudiendo requerirse un volumen total mayor $\mathrm{y} / \mathrm{o}$ una mayor concentración de glicerina.

En nuestro estudio no se observaron diferencias significativas en eventos adversos, pero tendieron a ser más favorables las cifras en el grupo tratado, especialmente en enterocolitis y hemorragia intracraneana, lo cual, al menos, da tranquilidad de que el procedimiento no aumenta estos riesgos. La asociación inversa entre más episodios de sepsis y la edad para alcanzar el aporte oral sugiere que el aporte en- 
teral se suspende o retrasa significativamente durante el cuadro infeccioso.

Según los estudios previos, la tolerancia alimentaria se puede estimular con aporte enteral mínimo precoz y mantenido, el uso de aporte continuo en el menor de $1.000 \mathrm{~g}$, el uso de leche materna, la administración de eritromicina, o usar una fórmula hidrolizada ${ }^{17}$. El uso de enemas como medida general en recién nacidos de extremo bajo peso de nacimiento no está apoyado por la evidencia, pero el uso en niños con retardo en eliminación de meconio y distención abdominal parece adecuado, con el eventual uso de Gastrografin ${ }^{\circledR}$ en la sospecha de impacto meconial que ha sido recomendado ${ }^{15,16,18}$.

\section{Referencias}

1.- Krasna IH, Rosenfeld D, Salerno P: Is it necrotizing enterocolitis, microcolon of prematurity, or delayed meconium plug? A dilemma in the tiny premature infant. J Pediatr Surg 1996; 31: 855-8.

2.- Emil S, Nguyen T, Sills J, Padilla G: Meconium obstruction in extremely low-birthweight neonates: guidelines for diagnosis and management. J Pediatr Surg 2004; 39: 731-7.

3.- Mihatsch W, Franz A, Linder W, et al: Meconium passage in extremely low birthweight infants and its relation to very early enteral nutrition Acta Paediatr 2001; 90: 409.

4.- Dimmitt RA, Moss RL: Meconium diseases in infants with very low birth weight. Semin Pediatr Surg 2000; 9: 79-83.

5.- Flidel-Rimon O, Friedman S, Lev E, Juster-Reicher A, Amitay M, Shinwell ES: Early enteral feeding and nosocomial sepsis in very low birthweight infants. Arch Dis Child F \& NEd 2004; 89: F289-92.

6.- Stoll BJ, Gordon T, Korones SB, et al: Late-onset sepsis in very low birth weight neonates: a report from the National Institute of Child Health and Human Development Neonatal Research Network. J Pediatr 1996; 129: 63-71.
7.- Koshinaga T, Gotoh H, Sugito K, et al: Spontaneous localized intestinal perforation and intestinal dilatation in very-low-birthweight infants. Acta Paediatr 2006; 95. 1385.

8.- Kubota A, Yamanaka H, Okuyama EH, et al: Focal intestinal perforation in extremely-low-birth-weightneonates: etiological consideration from histological findings. Pediatr Surg Int 2007 23: 997-1000.

9.- Sáez de Pipaón M, Montes MT, San José B, et al: Acquisition of full enteral feeds may depend on stooling pattern in very premature infants. J Perinat Med 2012; 40: 427-31.

10.- Garza-Cox $S$, Kenney $S$, Angel $C$, et al: Meconium Obstruction in the Very Low Birth Weight Premature Infant. Pediatrics 2004; 114: 285.

11.- Haiden N, Jilma B, Gerhold B, et al: Small Volume Enemas Do Not Accelerate Meconium Evacuation in Very Low Birth Weight Infants. J Ped Gastroenterol Nutr 2007; 44: 270.

12.- Shim S, Kim H, Kim D, et al: Induction of Early Meconium Evacuation Promotes Feeding Tolerance in Very Low Birth Weight Infants. Neonatology 2007; 92: 67.

13- Shah V, Chirinian N, Lee S: EPIQ Evidence Review Group. Does the use of glycerin laxatives decrease feeding intolerance in preterm infants? Paediatr Child Health 2011; 16 (9): e68-70.

14.- Khadr SN, Ibhanesebhor SE, Rennix C, et al: Randomized Controlled Trial: Impact of Glycerin Suppositories on Time to Full Feeds in Preterm Infants. Neonatology 2011; 100: 169-76.

15.- Haiden N, Norooz F, Klebermass-Schrehof K, et al: Preterm Infants The Effect of an Osmotic Contrast Agent on Complete Meconium Evacuation in Preterm Infants. Pediatrics 2012; 130; e1600.

16.- Emil S, Nguyen T, Sills J, Padilla G: Meconium obstruction in extremely low-birth-weight neonates: guidelines for diagnosis and management. J Pediatr Surg 2004; 39 (5): 731-73.

17.- Neu J, Zhang L: Feeding intolerance in very lowbirthweight infants: what is it and what can we do about it? Acta Paediatr Suppl 2005; 94: 93-9.

18.- Siddiqui MMF, Drewett M, Burge DM: Meconium obstruction of prematurity. Arch Dis Child Fetal Neonatal Ed. 2012; 97 (2): F147-50. 